May 1996

IASSNS-HEP 96/55

hep-ph/9605408

\title{
Experimental Consequences of a Minimal Messenger Model for Supersymmetry Breaking
}

\author{
K.S. Babu, Chris Kolda, and Frank Wilczek \\ School of Natural Sciences \\ Institute for Advanced Study \\ Princeton, NJ 08540
}

\begin{abstract}
We calculate the low-lying spectrum of new particles expected in a minimal model wherein supersymmetry breaking at $\lesssim 100 \mathrm{TeV}$ is indirectly transmitted to the Standard Model. We calculate the couplings of these particles relevant to their most nearly accessible experimental signatures, and estimate those signatures quantitatively. Running of various couplings plays a crucial role in the phenomenology, specifically in generating an adequate $\widetilde{N}_{1}-\widetilde{\ell}_{R}$ splitting.
\end{abstract}

\section{Introduction}

Since the widespread realization that successful unification of gauge couplings can be achieved in models including the minimal supersymmetric extension of the standard model (perhaps augmented with complete $S U(5)$ multiplets) [1], anticipation of the imminent discovery of direct evidence for real - as opposed to virtual - supersymmetry has become almost palpable. On the other hand, there has been no consensus regarding the form this discovery might take. Theoretical control over the many new parameters that arise even in minimal supersymmetric extensions of the standard model is not adequate to guide one through a bewildering number of choices that substantially affect the predictions for observable phenomena.

Recently a class of models that potentially enjoy great predictive power has been identified [2]. The leading idea of these models is that the fundamental breaking of supersymmetry occurs in a "hidden sector" and is characterized by a scale $\Lambda \lesssim 100 \mathrm{TeV}$. This breaking is supposed to be conveyed to our observable sector, including the minimal supersymmetric standard model, by a specific, highly symmetric interaction term

\footnotetext{
${ }^{1}$ Work supported in part by the Department of Energy contract No. DE-FG02-90ER40542 and by the Monell Foundation.

${ }^{2}$ E-mail: babu@sns.ias.edu, kolda@sns.ias.edu, wilczek@sns.ias.edu
} 
(to be discussed more precisely below). This is to be contrasted with the situation in a class of models, commonly referred to as supergravity models, that has been much more popular. In supergravity models the fundamental symmetry breaking scale is $\Lambda \sim 10^{9} \mathrm{TeV}$, and the hidden sector communicates with the observable sector by gravitational-strength interactions. A decisive phenomenological difference between these classes of models arises because of the different role played by the gravitino $\widetilde{G}$, which is essentially the Nambu-Goldstone fermion of spontaneous supersymmetry breaking. Its mass is generically given as $M_{\widetilde{G}}=\frac{\Lambda^{2}}{M_{\text {Planck }}}$, whereas its couplings to ordinary matter scale as $\frac{1}{\Lambda^{2}}$ [3]. In most supergravity models the gravitino is massive and very weakly coupled, and although it could be significant cosmologically, it plays no very direct role in ordinary phenomenology. In the (relatively) low energy messenger models the gravitino is exceedingly light, and although its couplings are very feeble they can be such as to cause the next lightest, photino-like particle $\widetilde{N}_{1}$ to decay via $\widetilde{N}_{1} \rightarrow \gamma+\widetilde{G}$ within a distance that might be difficult to resolve experimentally [4]. Generically, then, in these models one will find that pair-production of supersymmetric (R-odd) particles will lead eventually, perhaps after a cascade of decays, to final states containing $\gamma \gamma$ and missing energy. The recent observation [0] of a dramatic event with a final state containing hard $e^{+} e^{-} \gamma \gamma$ and missing transverse energy by the CDF collaboration therefore lends special interest to these models.

In this paper we shall calculate the low-lying spectrum and interactions in a minimal messenger model of the kind mentioned, in sufficient detail to estimate the most nearly accessible processes. We find a remarkably tight and specific pattern of consequences. In the course of the analysis we shall find that some apparent difficulties of the model are resolved by careful attention to the running of various couplings. Specifically, the tiny $\widetilde{N_{1}}-\widetilde{e_{R}}$ mass splitting one finds using bare couplings, and which if correct would render the interpretation of the observed $e^{+} e^{-} \gamma \gamma$ event problematic [6], is found to be greatly enhanced by their running (and by the inclusion of D-term effects). Similarly a possible difficulty with the apparent smallness of the $B \mu$ parameter, which if valid would imply the existence of a weak scale axion, is automatically repaired.

\section{Spectrum}

In the minimal messenger model (MMM) supersymmetry breaking is conveyed to the standard model particles by the matter fields $\left(q^{\prime}, \ell^{\prime}\right)+\left(\bar{q}^{\prime}, \bar{\ell}^{\prime}\right)$ having the quantum numbers $\mathbf{5}+\overline{\mathbf{5}}$ under $S U(5)$. These fields are assumed to acquire supersymmetry breaking masses through their couplings to a standard model singlet field $X$ which develops vacuum expectation values along its scalar as well as its $F$-components. Explicit models where this occurs dynamically have been constructed [2]. Typically these models contain the superpotential couplings

$$
W=\lambda_{q} \bar{q}^{\prime} q^{\prime} X+\lambda_{l} \bar{\ell}^{\prime} \ell^{\prime} X
$$


which renders a non-supersymmetric spectrum for the $\left(q^{\prime}, \ell^{\prime}\right)$ fields through $F_{X} \neq 0$. The $\left(q^{\prime}, \ell^{\prime}\right)$ fields, through their standard model gauge interactions, induce SUSY breaking masses for the R-odd scalars $(\widetilde{q}, \widetilde{\ell})$ and gauginos $(\widetilde{B}, \widetilde{W}, \widetilde{g})$. The gaugino mases arise through one-loop diagrams and are given (in the approximation $F_{X} \ll$ $\left.\lambda_{q, \ell}\langle X\rangle^{2}\right)$ as

$$
M_{i}(\Lambda)=\frac{\alpha_{i}(\Lambda)}{4 \pi} \Lambda
$$

Here $\Lambda \equiv F_{X} /\langle X\rangle$ is the effective SUSY breaking scale, which is close to the mass scale of the $\mathbf{5}+\overline{\mathbf{5}}$ fields. The scalar masses arise through two-loop diagrams and are given as

$$
m^{2}(\Lambda)=2 \Lambda^{2}\left\{C_{3}\left[\frac{\alpha_{3}(\Lambda)}{4 \pi}\right]^{2}+C_{2}\left[\frac{\alpha_{2}(\Lambda)}{4 \pi}\right]^{2}+\frac{3}{5}\left(\frac{Y}{2}\right)^{2}\left[\frac{\alpha_{1}(\Lambda)}{4 \pi}\right]^{2}\right\}
$$

We have adopted the usual $S U(5)$ normalization of the hypercharge coupling, $\alpha_{1} \equiv$ $\frac{5}{3} \alpha_{Y}$ and defined $Y$ as $Q=T_{3}+\frac{Y}{2}$. In Eq. (3), $C_{3}=4 / 3$ for colored scalars and zero for sleptons, $C_{2}=3 / 4$ for $S U(2)$ doublets and zero for singlets.

The mass relations of Eqs. (2)-(3) will arise in a large class of models which might differ in the details of supersymmetry breaking; it is sufficient that SUSY breaking is conveyed to the standard model particles through the $\mathbf{5}+\overline{\mathbf{5}}$ fields as in Eq. (1). Thus the mass relations would hold even if SUSY is broken spontaneously by the O'Raifearteigh mechanism ( $F$-type breaking). The $\mathbf{5}+\overline{\mathbf{5}}$ fields is of course the simplest extension of the standard model sector that preserves the successful unification of the gauge couplings.

The relations in Eqs. (2)-(3) imply that there is a clean separation of scales among the SUSY particles, as their masses scale with the interaction strength. Thus the squarks are the heaviest, followed by the gluino, left-handed sleptons $\left(\widetilde{\ell}_{L}\right), \widetilde{W}$, righthanded sleptons $\left(\widetilde{\ell}_{R}\right)$, and the $B$-ino, which is the next-to-lightest R-odd particle (the lightest is the gravitino $\widetilde{G}$ ). Their masses (neglecting RGE-running) are in the ratios $11.6: 7.0: 2.5: 2: 1.1: 1.0$. From an immediate experimental perspective the most interesting particles are the lightest ones, $\tilde{\ell}_{R}$, the chargino (which we shall denote by $\widetilde{W}$ ) and the two neutralinos $\left(\widetilde{N}_{1}\right.$ and $\left.\widetilde{N}_{2}\right)$. The latter are essentially pure gaugino eigenstates since their mixing with the Higgsinos are suppressed by the large $\mu$ parameter required in the model.

The relations in Eqs. (2) -(3) receive significant corrections from the renormalization group evolution. This evolution has several interesting consequences:

- It drives the mass-squared of $H_{u}$, the MSSM Higgs doublet that couples to the top quark, which is positive at the scale $\Lambda$, to a negative value near $M_{Z}$ and thus facilitates electroweak symmetry breaking. The mass-splitting between $H_{u}$ and $H_{d}$ is given by

$$
m_{H_{u}}^{2}-m_{H_{d}}^{2} \simeq-\frac{6 h_{t}^{2}}{8 \pi^{2}} m_{\widetilde{t}}^{2} \ln \left(\frac{\Lambda}{m_{\widetilde{t}}}\right)
$$




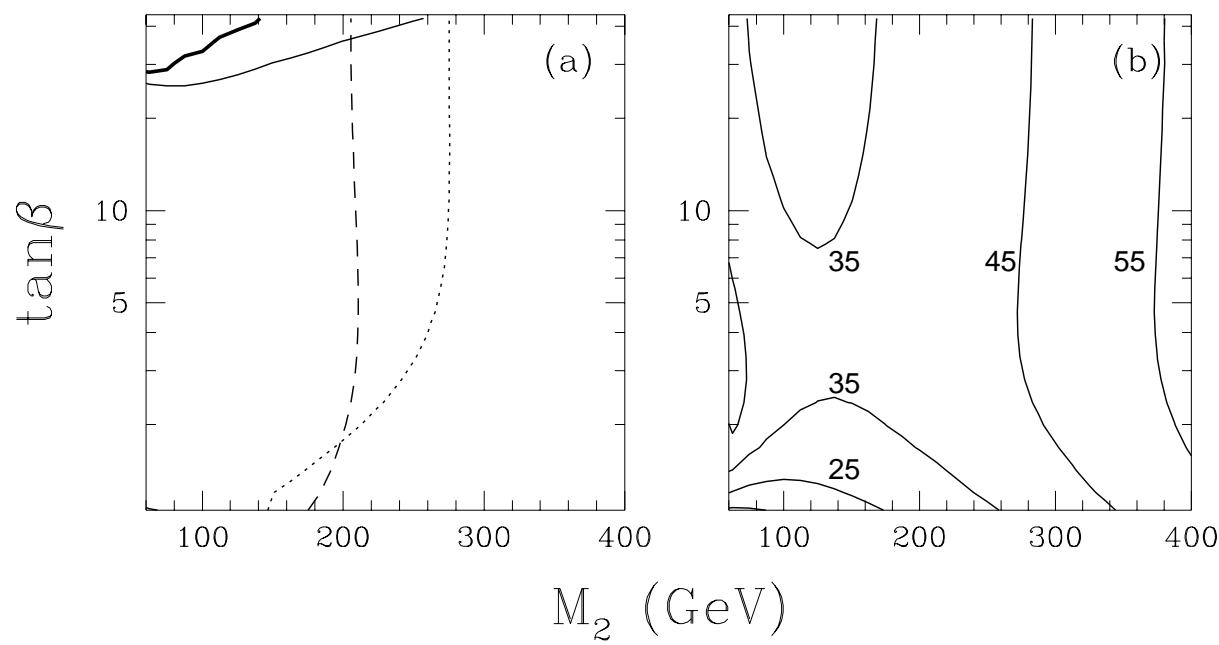

Figure 1: (a) Boundaries of interesting regions in the $\left(M_{2}, \tan \beta\right)$ plane: the region above the dark, solid line does not have a QED-preserving potential minimum; in the region above the light solid line, the $\widetilde{\tau}_{R}$ is the NLSP; in the region to the right of the dashed line, the decay $\widetilde{W} \rightarrow W \widetilde{N}_{1}$ is kinematically allowed; to the right of the dotted line the decay $\widetilde{N}_{2} \rightarrow \widetilde{N}_{1} h^{0}$ is kinematically allowed. (b) Contours of $m_{\widetilde{\ell}_{R}}-m_{\widetilde{N}_{1}}$ in $\mathrm{GeV}$, for $\mu<0$.

Observe that $H_{u}$ receives a mass correction proportional to $m_{\widetilde{t}}^{2}$, which is much larger than $m_{H_{u}}^{2}$.

- It lowers the masses of the $(\widetilde{B}, \widetilde{W})$, and raises the masses of $\widetilde{\ell}_{R}$ (as well as $\widetilde{\ell}_{L}$ and $\widetilde{q}$ ), from their bare values at $\Lambda$. Consequently, the mass splitting between $\widetilde{B}$ and $\widetilde{\ell}_{R}$ is enhanced at the weak scale. We find that a mass splitting of $10 \mathrm{GeV}$ at $\Lambda$ typically becomes $(20-25) \mathrm{GeV}$ at $M_{Z}$. Upon including, as well, the positive $D$-term correction to the mass of $\widetilde{\ell}_{R}$, the mass splitting becomes about $35 \mathrm{GeV}$ for $M_{2} \simeq 200 \mathrm{GeV}$. (For definitiveness we have taken $\Lambda=100 \mathrm{GeV}$ for the numbers given here.) Contours of this splitting are shown in Figure [1. This difference has significant impact on the interpretation of the observed eer $\gamma$ event at CDF.

- It supplies a sufficiently large mass to the would-be axion of the model. The soft bilinear mass term $B \mu$ for the Higgs field, $\left(B \mu H_{u} H_{d}+H . c.\right)$ in the Higgs potential, is extremely small at $\Lambda$. If not for the effect of running (and in the absence of other contributions to $B(\Lambda)$ ), one would find a weak scale axion, in conflict with observation. However, we find that a large enough $B$ is generated in the process of running so that $m_{A}^{2}=-2 B \mu / \sin 2 \beta \sim(400 \mathrm{GeV})^{2}$.

After including the effects of renomalization group evolution and D-terms, the mass spectrum of the model looks as follows. The gaugino masses obey a simple 
scaling with the the gauge couplings, so that

$$
M_{i}=M_{i}(\Lambda) \frac{\alpha_{i}}{\alpha_{i}(\Lambda)}
$$

The masses of $\tilde{\ell}_{R}, \tilde{\ell}_{L}$ and $H_{d}$ are given by

$$
\begin{aligned}
m_{\widetilde{\ell}_{L}, H_{d}}^{2}= & m_{\widetilde{\ell}_{L}, H_{d}}^{2}(\Lambda)+\frac{3}{2} M_{2}^{2}\left(\frac{\alpha_{2}^{2}(\Lambda)}{\alpha_{2}^{2}}-1\right)+\frac{1}{22} M_{1}^{2}\left(\frac{\alpha_{1}^{2}(\Lambda)}{\alpha_{1}^{2}}-1\right) \\
& -M_{Z}^{2}\left(\frac{1}{2}-s_{W}^{2}\right) \cos 2 \beta \\
m_{\widetilde{\ell}_{R}}^{2}= & m_{\widetilde{\ell}_{R}}^{2}(\Lambda)+\frac{2}{11} M_{1}^{2}\left(\frac{\alpha_{1}^{2}(\Lambda)}{\alpha_{1}^{2}}-1\right)-M_{Z}^{2} s_{W}^{2} \cos 2 \beta
\end{aligned}
$$

where the last terms are the $D$-term contributions, and should be omitted for $m_{H_{d}}^{2}$. The $A$ and $B$ parameters are given by

$$
\begin{array}{r}
A_{t} \simeq A_{t}(\Lambda)+M_{2}(\Lambda)\left[-1.85+0.94 \frac{Y_{t}}{Y_{f}}\right] \\
B \simeq B(\Lambda)-\frac{1}{2} A_{t}(\Lambda)+M_{2}(\Lambda)\left[-0.12+0.47 \frac{Y_{t}}{Y_{f}}\right],
\end{array}
$$

which hold for low to intermediate values of $\tan \beta$. Here $Y_{t}=h_{t}^{2}$ is the top Yukawa coupling squared, and $Y_{f} \simeq 2.79$. $A_{u, c}$ are obtained from $A_{t}$ by setting $Y_{t}=0$. The final mass ratios for $\widetilde{q}: \widetilde{g}: \widetilde{\ell}_{L}: \widetilde{W}: \widetilde{\ell}_{R}: \widetilde{N}_{1}$ are given by $9.3: 6.4: 2.6: 1.9: 1.35: 1.0$. (All of our numerical results are derived using the complete one-loop RGE's.)

In the minimal messenger model without any modification of the Higgs sector, one expects $A$ and $B$ parameters to be negligible at $\Lambda$, since they arise only from higher loops (see however Ref. [7]). However, in the process of running, significant $A$ and $B$ can be induced. Furthermore, in this constrained version, the parameter $\tan \beta$ and the sign of $\mu$ are determined. These results follow from minimizing the Higgs potential, which leads to

$$
\begin{aligned}
\sin 2 \beta & =\frac{-2 B \mu}{m_{H_{u}}^{2}+m_{H_{d}}^{2}+2 \mu^{2}} \\
\mu^{2} & =\frac{m_{H_{d}}^{2}-m_{H_{u}}^{2} \tan ^{2} \beta}{\tan ^{2} \beta-1}-\frac{1}{2} M_{Z}^{2} .
\end{aligned}
$$

The last relation implies that $\mu$ is large in the model. If $B(\Lambda)=0$, we can infer its effective value at $M_{Z}$. Tan $\beta$ is then determined through the RGE-improved tree-level potential of Eq. (9) to lie at $\tan \beta \approx 30$; estimates of the full 1-loop contributions appear to push $\tan \beta$ up to values near $\tan \beta \approx 40$ to 50 . In this most economical version, one also finds the sign of $\mu$ to be negative. 
A further constraint arises from demanding that the $\widetilde{\tau}_{R}$ mass-squared not turn negative (otherwise the vacuum will be superconducting). In Figure 11 we show the contour of $m_{\widetilde{\tau}_{R}}^{2}=0$ in the $\tan \beta-M_{2}$ plane; $\tan \beta \gtrsim 50$ is excluded. A more stringent constraint along these lines is to require, in the spirit of the phenomenology we are attempting to address, that $m_{\widetilde{\tau}_{R}}>m_{\widetilde{N}_{1}}$, otherwise $\widetilde{N}_{1}$ will decay dominantly into $\tau+\widetilde{\tau}_{R}$, destroying the $\gamma \gamma$ signature. The contour where $\widetilde{\tau}_{R}$ mass equals that of $\widetilde{N}_{1}$ is also shown in Figure 1; above that line the $\widetilde{\tau}_{R}$ is the lighter. Because the bound corresponds roughly to $\tan \beta \sim 25$, we shall focus only on the range $\tan \beta \leq 25$ in presenting our numerical results.

\section{Production and Decay of Sparticles}

Given the physical parameters $M_{2}, \tan \beta$ and $\operatorname{sgn} \mu$ all cross-sections and branching ratios within the MMM can be calculated. It is particularly important that within the MMM there are three widely separated mass scales: light particles with only $U(1)$ hypercharge interactions, intermediate mass particles with $S U(2)_{L}$ interactions, and heavy particles with $S U(3)_{C}$ interactions. The heavy states (squarks and gluinos) are typically around $1 \mathrm{TeV}$ for light particle masses consistent with LEP bounds; therefore their production rates even at the upgraded FNAL will be orders of magnitude below observable signal levels. Finally, the electroweak breaking constraint usually fixes $\mu$ such that $M_{2} \ll|\mu| \lesssim M_{3}$, pushing sparticles whose masses come from $\mu$ (i.e., higgsinos and the second doublet of Higgs bosons) out of the reach of current accelerators.

On the other hand, the light and intermediate mass sparticles can have nonnegligible production rates even in Run I of FNAL $\left(\int \mathcal{L} \simeq 120 \mathrm{pb}^{-1}\right)$. For this study of the MMM, we consider a $p \bar{p}$ collider/detector with $\sqrt{s}=1.8 \mathrm{TeV}$, as at FNAL Run I, with $100 \%$ efficiency for tagging all relevant final states. Because the sparticles are always produced in pairs, and because the gluinos and squarks are too heavy to be produced at reasonable $x$, we need only consider Drell-Yan (DY) production processes. Each process is calculated at the parton level for $\hat{s}=x_{1} x_{2} s$, then integrated over the proton parton distribution functions, $f_{q / p}\left(x_{i}\right)$, in this case using those of the CTEQ3M set [8]. Previous calculations of DY sparticle production were used or altered for the parton level processes studied here [9]. The parameter space studied is spanned by the three parameters $\left(M_{2}, \tan \beta, \operatorname{sgn} \mu\right)$ in the ranges $50 \leq M_{2} \leq 400$, $1<\tan \beta \leq 25$ and $\mu$ taking both signs.

There are a limited number of sparticle DY processes which can be expected to 
be observable at the current generations of hadron colliders:

$$
p \bar{p} \rightarrow Z \rightarrow\left\{\begin{array} { c } 
{ \widetilde { \nu } _ { \widetilde { \nu } ^ { * } } } \\
{ \widetilde { \ell } _ { L } \widetilde { \ell } _ { L } ^ { * } } \\
{ \widetilde { \ell } _ { R } \widetilde { \ell } _ { R } ^ { * } } \\
{ \widetilde { W } ^ { + } \widetilde { W } ^ { - } } \\
{ \widetilde { N } _ { i } \widetilde { N } _ { j } }
\end{array} \quad p \overline { p } \rightarrow W \rightarrow \left\{\begin{array}{c}
\widetilde{\ell}_{L} \widetilde{\nu}^{*} \\
\widetilde{W} \widetilde{N}_{i}
\end{array}\right.\right.
$$

where $i, j=1,2$. Not surprisingly, in order for production rates of the heavier slepton modes $\left(\widetilde{\nu} \widetilde{\nu}, \widetilde{\ell}_{L} \widetilde{\ell}_{L}\right)$ to be observable, rates for the lighter sparticles would be much larger, already providing an unambiguous signal at a collider. Since a large signal has in fact not been observed we will not consider the DY production of left-handed sleptons further.

One also expects (and we have explicitly verified) that the rate for double neutralino production $\left(\widetilde{N}_{i} \widetilde{N}_{j}\right)$ is small compared to the others, since in the limit $\widetilde{N}_{1}=\widetilde{B}^{0}$ and $\widetilde{N}_{2}=\widetilde{W}^{0}$ there are no $Z \widetilde{N}_{i} \widetilde{N}_{j}$ interaction terms in the MSSM. (This fact also implies that the calculation of $\widetilde{N}_{1}$ mass bounds from non-observation of $Z \rightarrow \widetilde{N}_{1} \widetilde{N}_{1}$ at LEP1.5 need not coincide with the kinematic limit of approximately $65 \mathrm{GeV}$; in fact we find the appropriate mass bound from OPAL 10 to be $10-15 \mathrm{GeV}$ below this limit.) Likewise, the $\widetilde{N}_{1} \widetilde{W}$ channel is suppressed due to the lack of a $W^{+} \widetilde{W}^{-} \widetilde{B}$ interaction. We find the production rate of $\widetilde{N}_{1} \widetilde{W}$ to be at least two orders of magnitude below the rates for competing processes, making it inaccessible at current colliders.

The dominant DY production channels are then $\widetilde{\ell}_{R} \widetilde{\ell}_{R}, \widetilde{N}_{2} \widetilde{W}$, and $\widetilde{W} \widetilde{W}$. In Figure 2 we have shown contours of cross-sections for the three production channels in the $\left(M_{2}, \tan \beta\right)$ plane, where we have summed over slepton flavors and gaugino charges. The contours are labelled in femtobarns (fb), and one should recall that $\sigma=10 \mathrm{fb}$ corresponds to 1 event in $100 \mathrm{pb}^{-1}$ of data. One notes some general features: (i) There is little or no dependence on $\operatorname{sgn} \mu$ in the most important channels, the largest dependence on sgn $\mu$ coming in the $\widetilde{W} \widetilde{N}_{1}$ channel, which is several orders of magnitude below those shown; (ii) the cross-sections are only weakly dependent on $\tan \beta$; (iii) at large $M_{2}$, the slepton production is largest, while it is the gaugino production which dominates at small $M_{2}$; in particular, when the cross-sections are near $10 \mathrm{fb}$, all three cross-sections are within a factor of four of each other; (iv) for $M_{2} \lesssim 160 \mathrm{GeV}$, the production rate for gauginos would be very large even now at the Tevatron.

The exact signature of each channel will depend on how its constituents decay. Consistent with the MMM and the CDF event, we take the gravitino to be the lightest R-odd particle, to which the next-lightest R-odd particle (the $\widetilde{N}_{1}$ ) decays via $\widetilde{N}_{1} \rightarrow \gamma \widetilde{G}$. Then essentially all sparticles produced will eventually decay to $\widetilde{N}_{1}$ and in turn to $\gamma+\mathbb{E}_{\perp}$; thus $\gamma \gamma+\mathbb{E}_{\perp}$ is a mandatory ingredient of the final state in all MMM sparticle production processes.

Within the MMM, the $\widetilde{\ell}_{R}=\widetilde{e}_{R}, \widetilde{\mu}_{R}$ and the $\widetilde{\tau}_{R}$ can only decay directly to the 2-body final state $\ell \widetilde{N}_{1}$ or $\tau \widetilde{N}_{1}$, where $\ell=e, \mu$ (we treat $\tau$ leptons separately for later convenience). Since the production cross-section for each flavor of slepton is the same, 


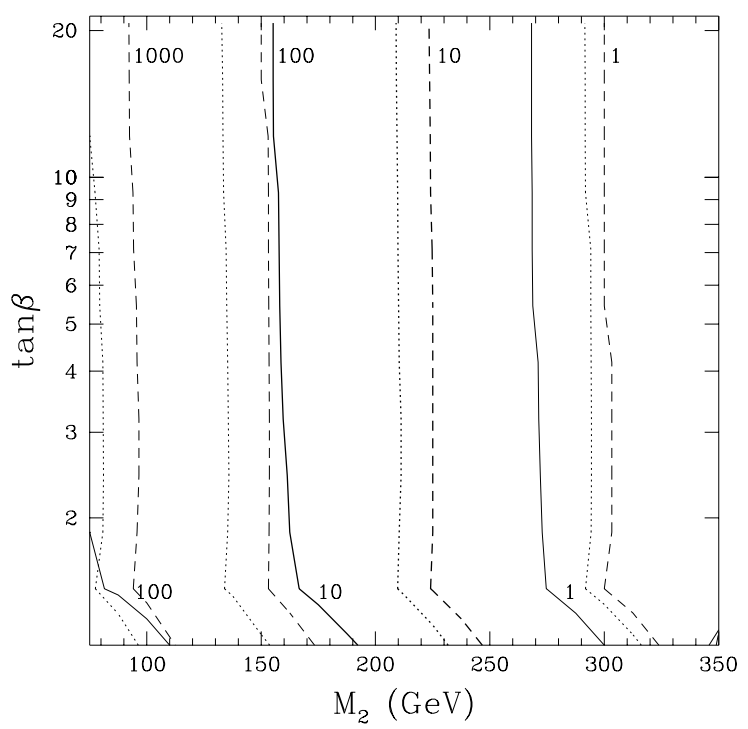

Figure 2: Cross-sections at the Tevatron for $\widetilde{\ell}_{R} \widetilde{\ell}_{R}$ (solid), $\widetilde{W} \widetilde{N}_{2}$ (dashed), and $\widetilde{W} \widetilde{W}$ (dotted) production. (Slepton flavors and gaugino charges summed over.) The contours are labelled in $\mathrm{fb}$; the labels at the top of the plot label both gaugino production channels, while those at the bottom label the slepton production. The contours for 1 event in $100 \mathrm{pb}^{-1}$ are in bold.

one observes a signal $\tau^{+} \tau^{-} \gamma \gamma+E_{\perp} 1 / 3$ of the time, and $\ell^{+} \ell^{-} \gamma \gamma+E_{\perp} 2 / 3$ of the time. Such a final state describes the ee $\gamma \gamma+\mathbb{E}_{\perp}$ event at CDF.

There has been some discussion in the literature as to whether the MMM could describe the CDF event through $\widetilde{\ell}_{R} \widetilde{\ell}_{R}$ production given the small mass splitting between $\widetilde{\ell}_{R}$ and $\widetilde{N}_{1}$ [6]. The authors of Ref. [6] were concerned because one of the electrons in the CDF event is highly energetic $\left(E_{\perp} \simeq 59 \mathrm{GeV}\right)$ while they assumed $m_{\widetilde{\ell}_{R}} \simeq 1.1 m_{\widetilde{N}_{1}}$, which in the mass range of interest corresponds to $\widetilde{\ell}_{R}-\widetilde{N}_{1}$ mass splitting of only about $10 \mathrm{GeV}$. The required boost for the initial pairs of sleptons seemed to reduce significantly the probability of interpreting this event as coming from MMM $\widetilde{\ell}_{R}$ production. This may be too pessimistic given the analysis of the $\widetilde{\ell}_{R}-\widetilde{N}_{1}$ mass difference in the previous Section. From that analysis, we find for the appropriate mass range that $m_{\widetilde{\ell}_{R}}-m_{\widetilde{N}_{1}} \simeq 35 \mathrm{GeV}$ and the required boost is small $(\gamma \simeq 1.3)$. However, as pointed out in [6], larger mass differences in turn imply smaller rates for $\widetilde{\ell}_{R} \widetilde{\ell}_{R}$ production with respect to the $\widetilde{W} \widetilde{N}_{2}$ and $\widetilde{W} \widetilde{W}$ channels. For a $35 \mathrm{GeV}$ mass splitting, this suppression is about a factor of two relative to a $10 \mathrm{GeV}$ splitting. Given the current paucity of data, this is completely consistent with observation.

Because $\widetilde{N}_{2}$ and $\widetilde{W}$ are nearly degenerate in mass, the preferred decay of the heavier of the two to the lighter via $\widetilde{W}^{ \pm} \rightarrow \widetilde{N}_{2} W^{ \pm}$, cannot occur. If $m_{\widetilde{W}}-m_{\widetilde{N}_{1}}>M_{W}$ then the 2-body decay $\widetilde{W}^{ \pm} \rightarrow \widetilde{N}_{1} W^{ \pm}$will be allowed. In Figure [1(a) the contour along which the decay becomes kinematically allowed is displayed. However, the coupling 
is suppressed by the lack of any $W^{ \pm} \widetilde{W}^{\mp} \widetilde{B}$ interaction in the MSSM, so unsuppressed 3 -body decays can compete with the 2-body mode. (Calculations of all relevant 2and 3-body decay widths exist in the literature [11].) The 3-body decays proceed through t-channel exchange of $\tilde{\ell}_{L}$ or $\widetilde{\nu}: \widetilde{W} \rightarrow \ell \nu_{\ell} N_{1}$. (In this way it might be possible to account for the $\mathrm{CDF}$ event using $\widetilde{W} \widetilde{W}$ production, without having to invoke coincidence against a $\frac{1}{9} \times \frac{1}{9}$ suppression in the $W$ decay branching ratios. A signal for this possibility is the existence of equal numbers of like and unlike lepton pair events, e.g., both $e^{+} e^{-} \gamma \gamma+\mathbb{E}_{\perp}$ and $\left.e^{+} \mu^{-} \gamma \gamma+\mathbb{E}_{\perp}.\right)$

At large $\tan \beta$ there is a strong enhancement in the 3-body decay into $\tau \nu_{\tau} \widetilde{N}_{1}$. This arises because at large $\tan \beta$ the $\tau$-Yukawa coupling becomes $\mathcal{O}(1)$ and couples to the $\widetilde{H}_{D}$ component of $\widetilde{W}$, which can be relatively large. For example, for $\mu$ positive (negative) and $\tan \beta$ greater than 5 (10), the branching ratio of $\widetilde{W} \rightarrow \tau \nu_{\tau} \widetilde{N}_{1}$ exceeds $50 \%$.

The decay channels of $\widetilde{N}_{2}$ are similarly a mix of 2-body decays suppressed by small couplings and 3-body decays suppressed by phase space. The three 2-body decays are to $\widetilde{N}_{1} h^{0}, \widetilde{N}_{1} Z$ and $\widetilde{\ell}_{R}^{ \pm} \ell^{\mp}$ final states. The first two can be either allowed or disllowed in alternative large regions of the parameter space; the third is always allowed. Each 2-body process suffers from small couplings which go to zero in the limit $\widetilde{N}_{2}=\widetilde{W}^{0}$ and $\widetilde{N}_{1}=\widetilde{B}^{0}$. For small gaugino-higgsino mixing the leading 2-body coupling is through the $\tau$-Yukawa coupling so that even at relatively small $\tan \beta$, decays to $\tau$ 's predominate.

When it is kinematically allowed, the decay $\widetilde{N}_{2} \rightarrow \widetilde{N}_{1} h^{0}$ has a large $(\sim 50 \%)$ branching fraction. Such a process could provide an interesting route toward pursuing Higgs physics at the Tevatron, with Higgs production neatly tagged by the hard photon pair. Because the lightest Higgs boson, $h^{0}$, receives large radiative corrections to its mass, there is considerable uncertainty about the precise prediction of $m_{h^{0}}$ for each point in our parameter space. Further, $m_{h^{0}}$ is sensitive to deviations from minimal messenger models, for example to inclusion of a singlet Higgs field (perhaps useful for generating a $\mu$-term). Therefore in presenting our results, we have pretended that the Higgs decay mode is disallowed; if it is allowed at all it is appreciable. We will highlight where applicable the swath through parameter space where the Higgs mode is open in the truly-minimal MMM. When on-shell Higgs production is disallowed, the coupling suppression serves to make any 3-body decay through the Higgs unobservably small. The contour along which the Higgs decay mode becomes accessible is shown in Figure 1 $1(\mathrm{a})$.

The two 3-body decays of $\widetilde{N}_{2}$ are via t-channel $\widetilde{\ell}_{L}$ and $\widetilde{\nu}: \widetilde{N}_{2} \rightarrow \ell^{+} \ell^{-} \widetilde{N}_{1}$ and $\widetilde{N}_{2} \rightarrow \nu \bar{\nu} \widetilde{N}_{1}$. At large $\tan \beta$ the $\tau \tau \widetilde{N}_{1}$ final state receives substantial enhancement from the $\widetilde{H}_{D}$ component of $\widetilde{N}_{2}$ coupling to $\widetilde{\tau}_{L} \tau$ via the large $\tau$-Yukawa coupling, as discussed in the case of $\widetilde{W}$ above. This means that for large $\tan \beta$, the production of $\widetilde{W}^{+} \widetilde{N}_{2}$ will give a signal of $\tau^{+} \tau^{+} \tau^{-} \gamma \gamma+\mathbb{E}_{\perp}$ nearly $100 \%$ of the time, while $\widetilde{W} \widetilde{W}$ production will look the same with one fewer final state $\tau$-lepton.

In Figure 3 we have folded the branching ratios into the production cross-sections 
to show the expected production rates for various sets of "final" states in the detector. Here we have defined on-shell $W^{ \pm}, Z$, and $h^{0}$ to be final states; the reader can identify the observed final states associated with production of these particles simply by multiplying by their well-known branching ratios. By keeping the gauge bosons in the final state, one also learns about the invariant masses of their decays products. Specifically, Figure 3 displays the $\sigma \cdot \mathrm{BR}$ of nine final states for $M_{2}=200 \mathrm{GeV}$ and varying $\tan \beta$. The two pairs of plots show the results for both signs of $\mu$.

Figure 3(a) shows the five most relevant channels at small $\tan \beta$ and $\mu>0$ (all channels that follow include $\left.\gamma \gamma+\mathbb{E}_{\perp}\right)$ : $\ell \ell$ (solid), $W \ell \ell$ (dotted), $W \tau \tau$ (short-dashed), $W W$ (long-dashed), and $W Z$ (dot-dashed). Figure 3(c) is the same for $\mu<0$. Figure 3(b) shows the four most relevant channels at large $\tan \beta$ and $\mu>0$ (not including $\ell \ell$ ): $\tau \tau \tau$ (solid), $\tau \tau$ (dotted), $\tau \ell \ell$ (short-dashed), and $W \tau$ (long-dashed). Figure $3(\mathrm{~d})$ shows the same five curves for $\mu<0$. In all four plots, the shaded region is the portion of parameter space that would allow the decay $\widetilde{N}_{2} \rightarrow \widetilde{N}_{1} h^{0}$.

From this analysis, it clearly emerges that if one is to interpret the CDF event to suggest $\sigma \times B R\left(p \bar{p} \rightarrow \widetilde{\ell}_{R} \widetilde{\ell}_{R}\right) \simeq(5-10) \mathrm{fb}$, then the MMM implies that there are several other channels which should also be accessible at the Tevatron. If $\tan \beta<5$ or 10 (depending on $\operatorname{sgn} \mu$ ), then the largest available channel is $W \ell \ell$ (for $\ell=e, \mu$ ) with $W$ decaying into 2 jets (2/3 of the time) or a single lepton ( $1 / 3$ of the time): $p \bar{p} \rightarrow \ell^{+} \ell^{-} j j \gamma \gamma+\mathbb{E}_{\perp}$ or $p \bar{p} \rightarrow \ell^{+} \ell^{-} \ell^{ \pm} \gamma \gamma+\mathbb{E}_{\perp}$. Second to this rate will be $W W \gamma \gamma+\mathbb{E}_{\perp}$ channels (with the usual $W$ decay branching ratios) and $W \tau \tau \gamma \gamma+\mathbb{E}_{\perp}$. There is also a small window around $3 \lesssim \tan \beta \lesssim 5$ and $\mu>0$ in which $W Z \gamma \gamma+\mathbb{E}_{\perp}$ is an important final state.

For large $\tan \beta, \tau$ production of all kinds dominates. If $\tan \beta \gtrsim 5(10)$, then the Tevatron should be producing $(n \tau) X \gamma \gamma+\mathbb{E}_{\perp}$ at rates comparable (or larger than) $e e \gamma \gamma+\mathbb{E}_{\perp}$, for $n=1,2,3$.

\section{Discussion}

We have described a minimal implementation of a low-energy messenger model for supersymmetry breaking. Outstanding features of the model include: generic production of final states including $\gamma \gamma$ and missing energy; clean separation of scales in the R-parity odd spectrum, with the squarks and gluinos much heavier than the electroweak gauginos and sleptons; and very small mixing of the $S U(2) \times U(1)$ neutralinos with Higgsinos and with each other. Many specific features are predicted in terms of very few parameters, so that the model could be rigorously tested, or eliminated, with quite a small amount of relevant data. If the $\mathrm{CDF} e^{+} e^{-} \gamma \gamma$ event represents a first indication, such data is not far beyond current reach.

Of course in insisting upon the absolutely minimal model we may have overreached. We have shown that some apparent difficulties with the minimal model are removed upon careful treatment of coupling renormalization and proper inclusion of D-terms. Nevertheless, one must remain open to such possibilities as different overall 

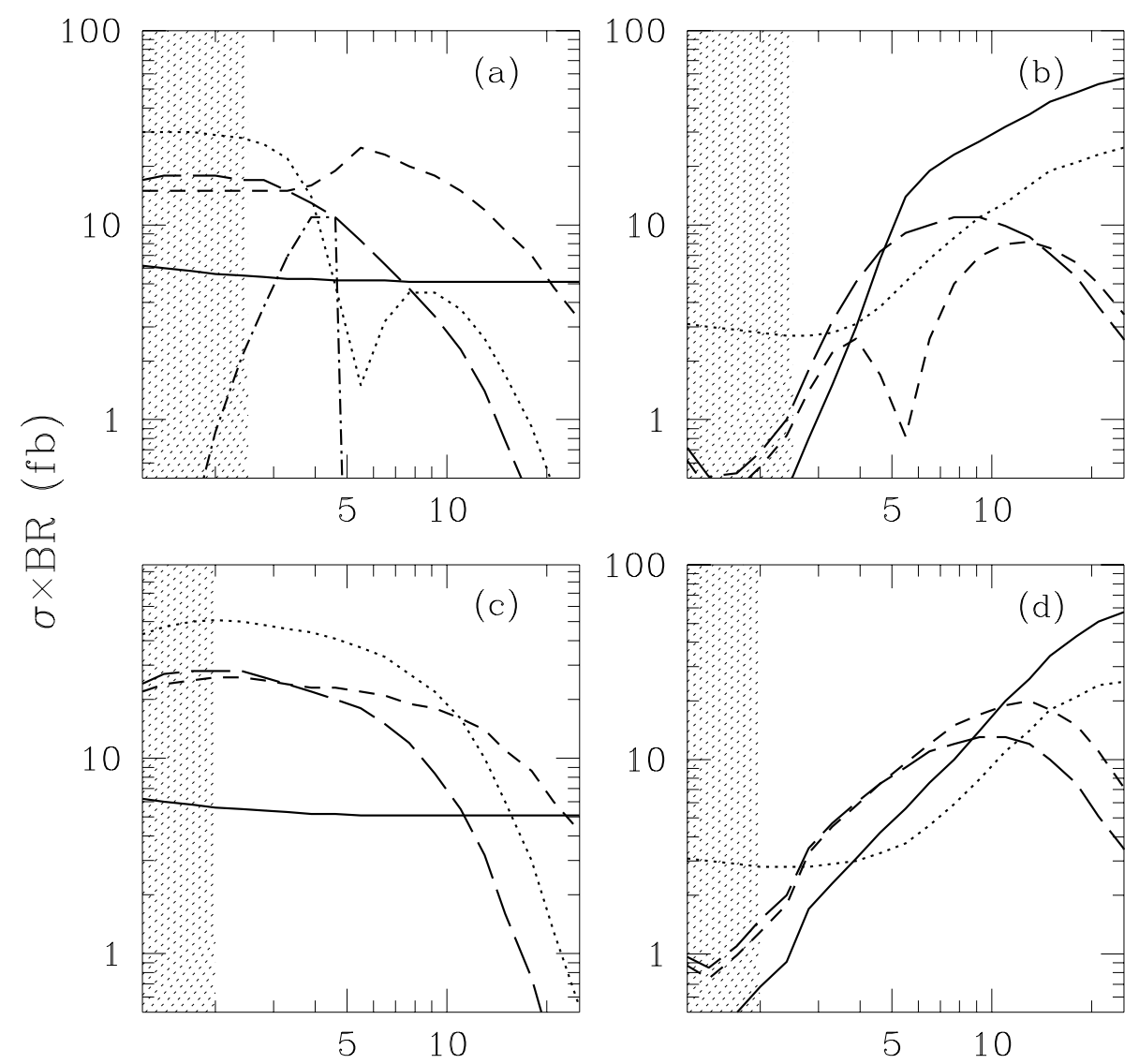

$\tan \beta$

Figure 3: Cross-section times branching ratio for $M_{2}=200 \mathrm{GeV}$ with $\tan \beta$ varied along the $\mathrm{x}$-axis. Both signs of $\mu$ are shown: (a)-(b) $\mu>0$, (c)-(d) $\mu<0$. The nine most relevant channels are displayed, divided between two subfigures for each $\operatorname{sgn} \mu$. The shading is in the region for which $\widetilde{N}_{2} \rightarrow \widetilde{N}_{1} h^{0}$ is allowed in the minimal model. See the text for a full description. 
mass scales for the sleptons as against the gauginos, or additional contributions to the parameters of the Higgs sector.

Finally let us briefly mention another possibility for physics capable of generating the $\mathrm{CDF} e^{+} e^{-} \gamma \gamma$ event, which if nothing else might serve as a useful foil. If there were heavy pion-like particles whose decay modes were dominantly $\Pi^{ \pm} \rightarrow W+\gamma$ and $\Pi^{0} \rightarrow Z Z$ or $Z \gamma$ or $\gamma \gamma$, their production and decay could induce many of the same final states as we have analyzed above, including the CDF event. The missing energy in this event would be carried off by neutrinos; but in other cases one might have $\gamma \gamma$ without significant missing energy, so that this alternative will be readily distinguishable.

\section{Acknowledgements}

We would like to thank J. Conway, H. Frisch, J. March-Russell and S. Treiman for many stimulating discussions.

\section{References}

[1] S. Dimopoulos, S. Raby and F. Wilczek, Phys. Today 44 (1991) 25, and references contained therein.

[2] M. Dine and A. Nelson, Phys. Rev. D48 (1993) 1277;

M. Dine, A. Nelson and Y. Shirman, Phys. Rev. D51 (1995) 1362;

M. Dine, A. Nelson, Y. Nir and Y. Shirman, Phys. Rev. D53 (1996) 2658.

[3] P. Fayet, Phys. Rep. 105 (1984) 21.

[4] S. Dimopoulos, M. Dine, S. Raby and S. Thomas, Phys. Rev. Lett. 76 (1996) 3494

S. Ambrosanio, G. Kane, G. Kribs, S. Martin and S. Mrenna, Phys. Rev. Lett. 76 (1996) 3498.

[5] S. Park, representing the CDF Collaboration, "Search for New Phenomena in CDF," in 10th Topical Workshop on Proton-Antiproton Collider Physics, ed. by R. Raha and J. Yoh (AIP Press, New York, 1995), report FERMILAB-CONF95/155-E.

[6] S. Dimopoulos, S. Thomas, and J. Wells, report SLAC-PUB-7148, hep-ph/9604452 (April 1996).

[7] G. Dvali, G. Giudice and A. Pomarol, report CERN-TH/96-61, hep-ph/9603238 (March 1996).

[8] H.L. Lai, et al, (CTEQ Collaboration), Phys. Rev. D51 (1995) 6139. 
[9] E. Eichten, I. Hinchliffe, K. Lane and C. Quigg, Rev. Mod. Phys. 56 (1984) 579; A. Bartl, H. Fraas and W. Majerotto, Nucl. Phys. B278 (1986) 1, Zeit. für Physik C30 (1986) 441.

[10] G. Alexander, et al, (OPAL Collaboration), report CERN-PPE/96-039 (March 1996).

[11] J. Gunion and H. Haber, Phys. Rev. D37 (1988) 2515;

A. Bartl, H. Fraas, and W. Majerotto, Zeit. für Physik C41 (1988) 475. 